\title{
The tilt effect in DOAS observations
}

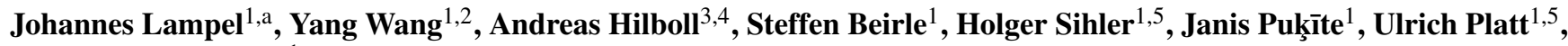 \\ and Thomas Wagner ${ }^{1}$ \\ ${ }^{1}$ Satellite Remote Sensing Group, Max Planck Institute for Chemistry, Mainz, Germany \\ ${ }^{2}$ Anhui Institute of Optics and Fine Mechanics, Chinese Academy Sciences, Hefei, China \\ ${ }^{3}$ Institute of Environmental Physics (IUP), University of Bremen, Bremen, Germany \\ ${ }^{4}$ Center for Marine Environmental Sciences (MARUM), University of Bremen, Bremen, Germany \\ ${ }^{5}$ Institute of Environmental Physics, University of Heidelberg, Heidelberg, Germany \\ a now at: Institute of Environmental Physics, University of Heidelberg, Heidelberg, Germany
}

Correspondence: Johannes Lampel (johannes.lampel@iup.uni-heidelberg.de) and Yang Wang (y.wang@mpic.de)

Received: 27 May 2017 - Discussion started: 3 July 2017

Revised: 11 October 2017 - Accepted: 31 October 2017 - Published: 12 December 2017

\begin{abstract}
Experience of differential atmospheric absorption spectroscopy (DOAS) shows that a spectral shift between measurement spectra and reference spectra is frequently required in order to achieve optimal fit results, while the straightforward calculation of the optical density proves inferior. The shift is often attributed to temporal instabilities of the instrument but implicitly solved the problem of the tilt effect discussed/explained in this paper.

Spectral positions of Fraunhofer and molecular absorption lines are systematically shifted for different measurement geometries due to an overall slope - or tilt - of the intensity spectrum. The phenomenon has become known as the tilt effect for limb satellite observations, where it is corrected for in a first-order approximation, whereas the remaining community is less aware of its cause and consequences.

It is caused by the measurement process, because atmospheric absorption and convolution in the spectrometer do not commute. Highly resolved spectral structures in the spectrum will first be modified by absorption and scattering processes in the atmosphere before they are recorded with a spectrometer, which convolves them with a specific instrument function. In the DOAS spectral evaluation process, however, the polynomial (or other function used for this purpose) accounting for broadband absorption is applied after the convolution is performed.

In this paper, we derive that changing the order of the two modifications of the spectra leads to different results. Assuming typical geometries for the observations of scattered sunlight and a spectral resolution of $0.6 \mathrm{~nm}$, this effect can be
\end{abstract}

interpreted as a spectral shift of up to $1.5 \mathrm{pm}$, which is confirmed in the actual analysis of the ground-based measurements of scattered sunlight as well as in numerical radiative transfer simulations. If no spectral shift is allowed by the fitting routine, residual structures of up to $2.5 \times 10^{-3}$ peak-topeak are observed. Thus, this effect needs to be considered for DOAS applications aiming at an rms of the residual of $10^{-3}$ and below.

\section{Introduction}

For a measured structured spectrum $s(\lambda)$ (e.g. scattered sunlight), the tilt effect emerges, because structures do not cancel out completely in the ratio of a measured spectrum $t(\lambda)$ relative to another spectrum with a different colour, which denotes the broadband spectral dependence. We show later that this can be interpreted as a spectral shift. This is due to the fact that the broadband shape of the atmospheric transmission and the convolution with the instrument function do not commute (Wenig et al., 2005).

The tilt effect was previously described and is explicitly corrected for by Sioris et al. (2003, 2004, 2006), Haley et al. (2004), Krecl et al. (2006), McLinden and Haley (2008), McLinden et al. (2010), and Rozanov et al. (2011) by including one additional tilt-effect pseudo absorber in the spectral analyses, the magnitude of which was determined from the spectral fit. In their pioneering work, Sioris et al. (2003) named this effect the "tilt effect" and corrected its impact by 
including a correction spectrum calculated using a radiative transfer model in the spectral analysis. However, Sioris et al. (2003) do not provide a mathematical derivation and instead estimates the effect's magnitude. It remains unclear whether this effect is related to spectral undersampling and if it is also significant for other observations of scattered sunlight. Haley et al. (2004) also provide formulae for the correction spectrum, state that the effect is directly related to spectral undersampling and note that the effect is stronger for $\mathrm{NO}_{2}$ retrievals in the blue spectral range than for $\mathrm{O}_{3}$ in the green spectral range due to smaller Fraunhofer lines. Rozanov et al. (2011) provide a mathematical derivation for the tilt effect correction spectrum (in their Appendix B) and state that the tilt effect can be interpreted as a spectral shift. However, also here, only one fixed correction spectrum is used, which is scaled accordingly with the fitting routine.

We will derive the tilt correction as an interpretation of a spectral shift for Gaussian instrument functions, which can, however, also vary with wavelength. We show that the calculated spectral shifts due to the tilt effect agree with the observed shifts from DOAS analyses of ground-based measurements.

We observed that for Multi Axis Differential Optical Absorption Spectroscopy (MAX-DOAS) evaluations (e.g. Hönninger and Platt, 2002), which allow for a spectral shift of the measurement spectrum relative to a reference spectrum, systematic spectral shifts of up to $2 \mathrm{pm}$ at a spectral resolution of the instrument of $0.6 \mathrm{~nm}$ are observed as shown in Fig. 2 with the exact magnitude depending on the observation geometry. If no spectral shift is allowed in the fitting routine, residual structures of up to $2.5 \times 10^{-3}$ peak-to-peak are observed (see Fig. 5). Thus, this effect needs to be considered for any DOAS application using a structured light source (such as the Sun), aiming at an rms of the residual of $10^{-3}$ and below. This is done implicitly in many DOAS retrieval codes by allowing for a spectral shift between measurement spectra and reference spectra. This option was originally introduced into the different analysis software to account for real shifts caused by instrumental instabilities (compare e.g. Peters et al., 2017). In fact, the observed shifts derived from the spectral analysis were usually attributed to such instrumental stabilities only.

MAX-DOAS instruments typically contain thermally stabilized spectrometers in order to avoid changes in their pixelto-wavelength calibration. For such instruments, the spectral stability within 1 day often has a similar magnitude to the tilt effect (often less than a few picometres). So-called Fraunhofer reference spectra are recorded regularly at zenithviewing direction: these are used as reference for the spectral analysis. If Fraunhofer reference spectra are recorded 1015 min each, then the change in spectral calibration of the instrument for the measurement spectrum relative to the Fraunhofer reference spectrum becomes small (typically $<0.1 \mathrm{pm}$, cf. Fig. 2) and no larger spectral shifts in the DOAS analysis can be explained by instrumental instabilities any more.
However, significant spectral shifts are still observed and are furthermore related to the telescope elevation angle of the MAX-DOAS observation. These can be explained in such cases by the tilt effect as shown in Sect. 3.4.

When a measured spectrum is evaluated relative to another spectrum of the same set-up, instrumental effects on the tilt are expected to cancel out, as both spectra are influenced in the same way, e.g. by the efficiency of the grating and the detector. However, if a measured spectrum is evaluated relative to a so-called Kurucz Sun spectrum (as e.g. in Burton and Sawyer, 2016; Lübcke et al., 2016), the instrumentally induced tilt change can also lead to an apparent relative spectral shift.

Another interesting aspect is that correction of the measured shifts including the tilt effect will allow the spectral stability of passive DOAS instruments to be estimated more precisely as shown in Sect. 3.4.

In Sect. 2, we mathematically derive the expected spectral shift for a simplified instrument function. The expected spectral shifts are compared in Sect. 3 to field measurements. For these, as in Sect. 4 for the case of synthetic spectra, good agreement is found. Finally in Sect. 5 we discuss different ways of how the tilt effect can be corrected. We provide examples and estimate its impact on the spectral retrieval.

\section{Mathematical derivation}

\subsection{Principle}

A sketch of the principle of the tilt effect is shown in Fig. 1 where two individual $\delta$-shaped emission lines are used instead of a Sun spectrum.

Two emission lines $\delta_{1}$ and $\delta_{2}$ at 349.8 and $350.2 \mathrm{~nm}$ are observed using a (virtual) spectrometer with a spectral resolution of $0.6 \mathrm{~nm}$. These are shown as Gaussian peaks ( $p 1$ and $p 2$ ) around each of the lines (grey areas). If both lines have the same intensity, the resulting total observed intensity (blue) has its maximum in the middle of the two lines at $350 \mathrm{~nm}$. If the lines are attenuated by the polynomial $p(\lambda)$ (drawn in red, in intensity space), the resulting total observed intensity (green) appears to be shifted in wavelength by $\Delta \lambda=-0.08 \mathrm{~nm}$. The unrealistically steep broadband slope in $p(\lambda)$ was chosen to illustrate the effect: typically the slope of the polynomial in intensity space in DOAS observations is 2 orders of magnitudes smaller, as is the spectral shift due to the tilt effect (see Sect. 3).

\subsection{Definitions}

The instrument response function or instrument slit function $h\left(\lambda_{0}, \lambda\right)$ describes the response of the spectrometer for incoming radiation of wavelength $\lambda_{0}$ at the response wavelength $\lambda$ on the detector. 


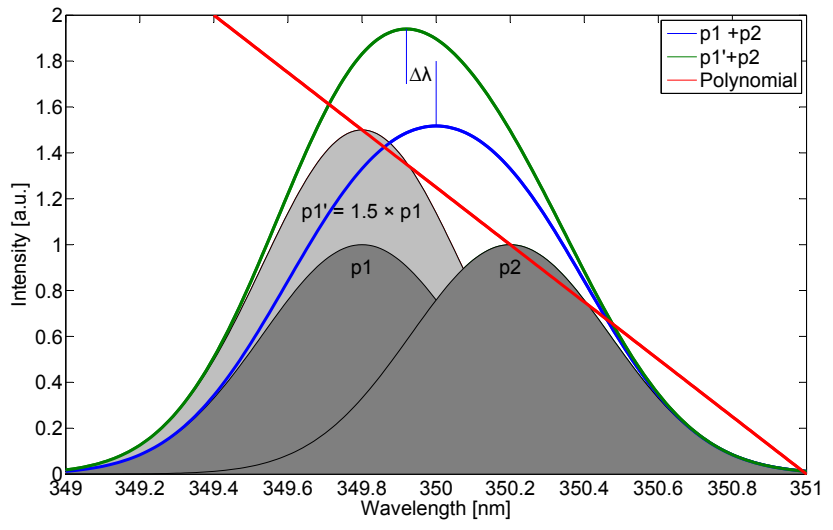

Figure 1. Illustration of the tilt effect: an explanation is found in Sect. 2.1.

Let $p(\lambda)$ be a polynomial in intensity space describing the broadband change in the shape of the spectrum due to scattering processes and broadband absorption in the atmosphere.

Finally $k(\lambda)$ is a high-resolution Sun spectrum, e.g. from Chance and Kurucz (2010).

A low-resolution Sun spectrum $s(\lambda)$ can be calculated from these quantities, where $\otimes$ denotes the convolution operator. For simplicity, $s(\lambda)$ is assumed to be direct sunlight with neither extinction nor absorption.

$s(\lambda)=k(\lambda) \otimes h\left(\lambda_{0}, \lambda\right)=\int \mathrm{d} \lambda_{0} h\left(\lambda_{0}, \lambda\right) k\left(\lambda_{0}\right)$,

and with the wavelength-dependent attenuation $p(\lambda)$, we obtain

$$
\begin{aligned}
t(\lambda) & =(k(\lambda) p(\lambda)) \otimes h\left(\lambda_{0}, \lambda\right) \\
& =\int \mathrm{d} \lambda_{0} h\left(\lambda_{0}, \lambda\right) p\left(\lambda_{0}\right) k\left(\lambda_{0}\right) .
\end{aligned}
$$

The optical density which is typically fitted in DOAS applications (Platt and Stutz, 2008) is then

$\tau(\lambda)=\ln \frac{t(\lambda)}{s(\lambda)}$.

The tilt effect in the current literature describes the fact that absorption structures in $s(\lambda)$ and $t(\lambda)$ (Fraunhofer lines and atmospheric absorbers on Earth) do not cancel out completely when calculating the optical depth $\tau$, even if $p(\lambda)$ is smooth. We will show that it produces an apparent shift $\Delta \lambda$ of $t(\lambda)$ with respect to $s(\lambda)$. It is caused by the broadband spectral variation $p(\lambda)$, typically approximated by a polynomial in optical density, which does not commute with the convolution with the instrument function $p(\lambda) s(\lambda)-t(\lambda) \neq$ 0 . In the next subsection, we therefore want to show the following equation:

$p(\lambda) s(\lambda) \stackrel{!}{\approx} t(\lambda-\Delta \lambda)=t(\lambda)-\Delta \lambda \frac{\partial t(\lambda)}{\partial \lambda}+\mathcal{O}\left(\Delta \lambda^{2}\right)$.
Apart from the shift $\Delta \lambda$, higher orders $\mathcal{O}\left(\Delta \lambda^{2}\right)$ are neglected here. Note that the right-hand side of Eq. (4) is similar to the tilt definition in Haley et al. (2004, their Eq. 20), even though it is not directly connected to a spectral shift there.

\subsection{Derivation}

Without restriction of generality, a Gaussian instrument function is used in the following, as it has some useful analytical properties. Here $\sigma$ is the standard deviation and $\lambda_{0}$ the centre wavelength.

$g\left(\lambda_{0}, \lambda\right)=\frac{1}{\sigma \sqrt{2 \pi}} e^{-\frac{\left(\lambda-\lambda_{0}\right)^{2}}{2 \sigma^{2}}}$

Note that any instrument function can be represented by a sum of Gaussian functions (Sect. 5.4) and that many instrument functions are indeed close to Gaussian shape, as in Beirle et al. (2017). To show a useful relation which is needed later, we set $\lambda_{0}=0$ for simplicity and we use a first-order polynomial

$q(\lambda)=1-w \lambda$.

We use $\frac{\partial g(\lambda)}{\partial \lambda}=-\frac{\lambda}{\sigma^{2}} g(\lambda)$ to reformulate

$q(\lambda) g(\lambda)=g(\lambda)-w \lambda g(\lambda)=g(\lambda)+w \sigma^{2} \frac{\partial g(\lambda)}{\partial \lambda}$,

which then is $g(\lambda+\Delta \lambda)+\mathcal{O}\left(\Delta \lambda^{2}\right)$ with $\Delta \lambda=w \sigma^{2}$. We find that the spectral shift $\Delta \lambda$ is indeed proportional to the product of the tilt $w$ of the spectrum and the square of the width of the instrument function. $\mathcal{O}\left(\Delta \lambda^{2}\right)$ represents second-order effects. This can also change of the effective shape of the instrument function (see Sect. 5.3).

The average $w$ from Eq. (6) (or later $\frac{\partial}{\partial \lambda} d(\lambda)$ with the DOAS polynomial $d(\lambda)$ from Eq. 13) was found within $0.025-0.01 \mathrm{~nm}^{-1}$ averaged over the fit interval using a fixed Fraunhofer reference spectrum for the MAD-CAT campaign described in Sect. 3.

For measurements, $p(\lambda)$ is normally not linear in $\lambda$ due to the characteristics of Mie and Rayleigh scattering. Therefore the derivative $\frac{\partial}{\partial \lambda} p(\lambda)$ is not constant and the spectral shift $\Delta \lambda$ also depends on the wavelength $\lambda$ itself.

With Eq. (7) we can calculate $t(\lambda)$ from Eq. (2):

$$
\begin{aligned}
t(\lambda) & =(k(\lambda) p(\lambda)) \otimes g\left(\lambda_{0}, \lambda\right) \\
& =\int \mathrm{d} \lambda_{0} g\left(\lambda_{0}, \lambda\right) p\left(\lambda_{0}\right) k\left(\lambda_{0}\right) .
\end{aligned}
$$

Taylor expansion of $p\left(\lambda_{0}\right)$ around $\lambda$ yields

$$
\begin{aligned}
t(\lambda)= & \int \mathrm{d} \lambda_{0} g\left(\lambda_{0}, \lambda\right)\left[p(\lambda)+\left.\left(\lambda_{0}-\lambda\right) \frac{\partial}{\partial \lambda^{\prime}} p\left(\lambda^{\prime}\right)\right|_{\lambda^{\prime}=\lambda}\right. \\
& \left.+\mathcal{O}\left(\lambda_{0}-\lambda\right)^{2}\right] k\left(\lambda_{0}\right),
\end{aligned}
$$


which is, with the shift from Eq. (7) at wavelength $\lambda$ and neglecting higher-order terms:

$$
\begin{aligned}
& \approx p(\lambda) \int \mathrm{d} \lambda_{0} g\left(\lambda_{0}, \lambda+\Delta \lambda(\lambda)\right) k\left(\lambda_{0}\right) \\
& \quad=p(\lambda) s(\lambda+\Delta \lambda(\lambda))
\end{aligned}
$$

with

$$
\Delta \lambda(\lambda)=\sigma^{2} \frac{1}{p(\lambda)} \frac{\partial}{\partial \lambda} p(\lambda)=\sigma^{2} \frac{\partial}{\partial \lambda} \ln (p(\lambda))
$$

$p(\lambda)$ is defined in intensity space and is related to the DOAS polynomial $d(\lambda)$ in optical density space (logarithm of intensity) via

$p(\lambda)=e^{-d(\lambda)}$.

We get

$\Delta \lambda(\lambda)=-\sigma^{2} \frac{\partial}{\partial \lambda} d(\lambda)$.

This is the more general case of Eq. (7) for non-linear DOAS polynomials.

\subsection{Relation to undersampling}

Previously the tilt effect was also associated with spectral undersampling (Chance et al., 2005): as, for example, described in Slijkhuis et al. (1999) for spectral data from satellite, a spectral shift between the observed measurement and reference spectra was introduced in order to correct for Doppler shifts between them. As these shifts (typically $<5 \mathrm{pm}$ ) are small compared to the spectral resolution of the instrument (typically $\approx 0.5 \mathrm{~nm}$ ), the spectral shift can be linearized and directly calculated from a high-resolution Sun spectrum (such as Chance and Kurucz, 2010) in order to also include artefacts of spectral undersampling. In Slijkhuis et al. (1999) the DOAS fit finally contained this linearized shift as well as the non-linear shift and squeeze parameters of the measurement spectrum relative to the reference spectrum. Also, the tilt effect introduces a spectral shift of similar magnitude and is corrected (in first-order approximation) in the same way. This potentially led to confusion in the available literature. The derivation of the tilt effect shown above does, however, not depend at all on the properties of the spectral binning of the instrument and can therefore be considered independent of the undersampling effects.

\subsection{Relation to the colour index}

The colour index $\operatorname{CI}\left(\lambda_{a}, \lambda_{b}\right)$ is defined by the ratio of intensities $I_{a}$ and $I_{b}$ at two distinct wavelengths $\lambda_{a}$ and $\lambda_{b}$ (see e.g. Sarkissian et al., 1991) and can be used to describe the tilt of a spectrum in first-order approximation.

$\mathrm{CI}\left(\lambda_{a}, \lambda_{b}\right)=\frac{I_{a}}{I_{b}}$
Instead of analysing the DOAS polynomial, it is often sufficient to look at the difference in colour indices of measurement spectra and reference spectra, or in other words, at the tilt of the spectrum (or part of a spectrum) as in Sioris et al. (2003). For a measurement spectrum $I^{\prime}$ with a DOAS polynomial $d(\lambda)$ relative to the reference spectrum $I$, we get

$\mathrm{CI}^{\prime}\left(\lambda_{a}, \lambda_{b}\right)=\frac{I_{a} e^{-d\left(\lambda_{a}\right)}}{I_{b} e^{-d\left(\lambda_{b}\right)}}$,

and thus we obtain using Eq. (11)

$$
\begin{aligned}
\mathrm{CI}^{\prime}-\mathrm{CI} & =\frac{I_{a}\left(e^{-d\left(\lambda_{a}\right)}-e^{-d\left(\lambda_{b}\right)}\right)}{I_{b} e^{-d\left(\lambda_{b}\right)}} \\
& =\left.\frac{I_{a}}{I_{b}} \frac{\lambda_{a}-\lambda_{b}}{e^{-d\left(\lambda_{b}\right)}} \frac{\partial}{\partial \lambda} e^{-d(\lambda)}\right|_{\lambda=\lambda_{c}} ; \lambda_{c} \in\left[\lambda_{a}, \lambda_{b}\right] \\
& \approx-\left.\frac{I_{a}\left(\lambda_{a}-\lambda_{b}\right)}{I_{b}} \frac{\partial}{\partial \lambda} d(\lambda)\right|_{\lambda=\lambda_{c}} ; \lambda_{c} \in\left[\lambda_{a}, \lambda_{b}\right] .
\end{aligned}
$$

This means that the difference in colour indices between different spectra is proportional to the derivative of the DOAS polynomial at a certain point $\lambda_{c}$ within the fit interval. The derivative of the DOAS polynomial is again proportional to the apparent spectral shift due to the tilt effect (Eq. 13). As an example the colour indices for 1 day of MAX-DOAS measurements are shown in Fig. $2 b$.

\section{Measurements}

For a spectral resolution of $\approx 0.6 \mathrm{~nm}$ and typical DOAS polynomials, shifts of up to around $1 \mathrm{pm}$ are expected using Eq. (13). In this section, we will set the expected spectral shift due to the tilt effect in relation to the spectral shift of the measurement spectrum in DOAS fits.

\subsection{Measurement site}

The Multi Axis DOAS - Comparison campaign for Aerosols and Trace gases (MAD-CAT) in Mainz, Germany took place on the roof of the Max Planck Institute for Chemistry (MPIC) during June and July $2013^{1}$. The measurement site is located on the outskirts of Mainz and is close to Frankfurt as well as several smaller towns. Eleven research groups participated with the MAX-DOAS instruments. The intercomparison is aimed primarily at the spectral retrieval of nitrogen dioxide $\left(\mathrm{NO}_{2}\right)$, formaldehyde ( $\left.\mathrm{HCHO}\right)$, nitrous acid ( $\mathrm{HONO}$ ) and glyoxal (CHOCHO), their azimuthal distributions and the retrieval of their respective vertical concentration profiles. Data from this campaign have been already published, e.g. in Ortega et al. (2015), Lampel et al. (2015), Peters et al. (2017) and Wang et al. (2017).

\footnotetext{
${ }^{1}$ http://joseba.mpch-mainz.mpg.de/mad_cat.htm
} 

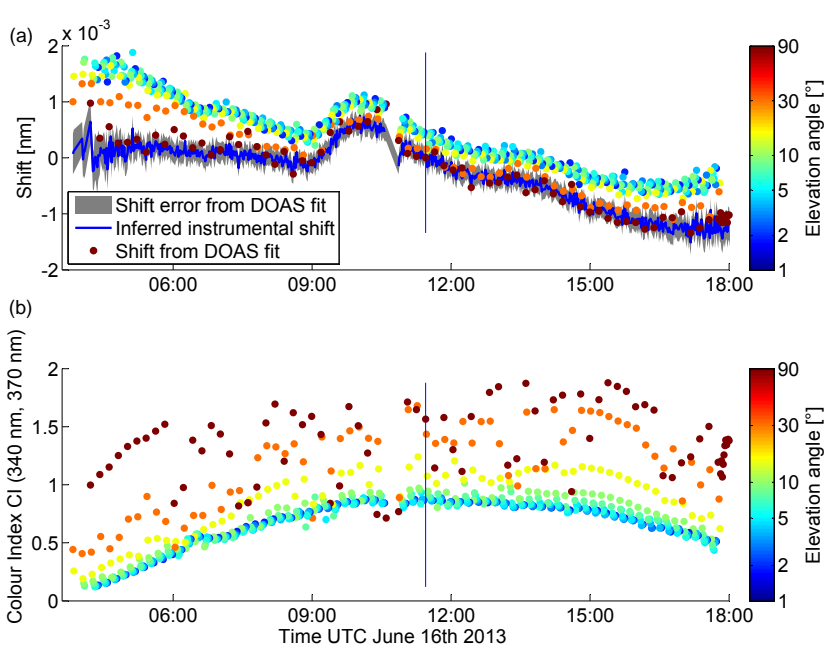

Figure 2. Measured shift (a) and colour index (340, $370 \mathrm{~nm})$ (b) as a function of time and observation elevation (colour coded) for 1 day (16 June 2013) during the MAD-CAT campaign relative to a Fraunhofer reference spectrum recorded close to local noon (thin blue vertical line). The thick blue line in the upper panel represents the pure instrumental shift after the shift introduced by the tilt effect was removed (see text).

\subsection{Instrument description}

We apply data obtained by an EnviMeS ${ }^{2}$ MAX-DOAS instrument during the MAD-CAT campaign. It is based on two Avantes ultra-low stray-light AvaSpec-ULS2048x64 spectrometers $(f=75 \mathrm{~mm})$ using a back-thinned Hamamatsu S11071-1106 detector. The spectrometer is temperature stabilized at $20^{\circ} \mathrm{C}$ with deviations of $\Delta T<0.02^{\circ} \mathrm{C}$ at the temperature sensor. The UV spectrometer covered a spectral range of $294-458 \mathrm{~nm}$ at a FWHM spectral resolution of $\approx 0.6 \mathrm{~nm}$ or $\approx 7$ pixels. The spectral stability was determined from the position of the Ca lines at around 393 and $397 \mathrm{~nm}$ and was typically better than $\pm 2 \mathrm{pm}$ per day and better than $\pm 5 \mathrm{pm}$ for the duration of the measurements from 6 June 2013 to 31 July 2013.

Mercury discharge lamp spectra recorded at different spectrometer temperatures yield a shift of the spectral calibration of this spectrometer type of about $4.5 \mathrm{pm} \mathrm{K}^{-1}$. The maximum deviation of the spectrometer temperature from the nominal temperature was $\Delta T<0.02 \mathrm{~K}$; thus less than $0.1 \mathrm{pm}$ spectral shift can be attributed to temperature instability close to thermal equilibrium under ideal conditions.

During laboratory test measurements, a change of the spectral calibration of the instrument over time was found to be proportional to the temperature difference outside the thermally insulated spectrometer box and the spectrometer temperature and is therefore attributed to the residual temperature differences due to heat flux from the Peltier element

\footnotetext{
${ }^{2}$ now continued by Airyx GmbH, Eppelheim, Germany, http:// www.airyx.de
}

through the spectrometer and the thermal insulation. We assume that this is the main reason for the variation of the inferred instrumental spectral shift in Fig. 2, which was already corrected for the shift introduced by the tilt effect. This observation later led to an improved mechanical set-up of the spectrometer box to reduce these internal temperature differences.

Mercury discharge lamp spectra used to obtain the instrument slit function $h\left(\lambda_{0}, \lambda\right)$ were recorded manually. No significant change of the instrument slit function shape was observed during the campaign.

The 1-D-telescope unit measures its elevation angle constantly using a MEMS acceleration sensor to determine the true vertical direction and corrects the elevation angle when it deviates from the nominal elevation angle. It has a vertical and horizontal field of view (FOV) of 0.2 and $0.8^{\circ}$. During daylight, spectra were recorded for 1 min each at 11 elevation angles of $90^{\circ}$ (zenith), 30, 15, 10, 8, 6- $1^{\circ}$ as long as solar zenith angles (SZA) were smaller than $87^{\circ}$. Until a SZA of $100^{\circ}$ zenith sky spectra were recorded at $90^{\circ}$ telescope elevation. The exposure time was adjusted within the DOASIS (Kraus, 2006) measurement script to obtain spectra at a typical saturation of $50 \%$.

\subsection{Analysis}

Even though the tilt effect is a general effect and not restricted to a certain wavelength range, here we adapted the HONO retrieval settings suggested by Wang et al. (2017) for the spectral analysis (see Table 1). Similar results were obtained in other wavelength intervals (e.g. a glyoxal retrieval window from 432 to $458 \mathrm{~nm}$ ).

The analysis of measured and synthetic spectra (see Sect. 4) was done using the DOASIS software using a noon Fraunhofer reference spectrum. The literature cross sections were convolved using the measured instrument slit function at $334 \mathrm{~nm}$.

\subsubsection{Shift and squeeze parameters}

The shift $a$ and squeeze $b$ (also called stretch) allow the DOAS fit to shift and squeeze cross sections in order to minimize the fit rms and compensate for instrumental instabilities and other factors which can influence the spectral calibration of the instrument.

This is parameterized typically in the following way:

$$
\Delta \lambda_{\text {shift }}(\lambda)=a+b\left(\lambda-\lambda_{0}\right)+c\left(\lambda-\lambda_{0}\right)^{2}+\mathcal{O}\left(\left(\lambda-\lambda_{0}\right)^{3}\right) .
$$

Higher orders such as $c$ are often not used and set to zero.

The choice of $\lambda_{0}$ depends on the implementation. It is the minimum wavelength of the fit interval in DOASIS and the middle of the fit interval in the QDOAS software package (Danckaert et al., 2012). Choosing $\lambda_{0}$ in the middle of the fit range has the advantage that the corresponding base func- 
Table 1. Retrieval wavelength intervals and reference spectra for the MAX-DOAS. $S_{0}$ denotes the SCD used for the $I_{0}$ correction during convolution, if applicable.

\begin{tabular}{|c|c|c|c|c|c|}
\hline & $T$ & $S_{0}$ & HONO & & \\
\hline Wavelength interval (nm) & End & & 373 & & \\
\hline $\mathrm{O}_{4}$ & $293 \mathrm{~K}$ & & $x$ & & Thalman and Volkamer (2013) \\
\hline $\mathrm{O}_{3}$ & $223 \mathrm{~K}$ & $1 \times 10^{18}$ molec cm $^{-2}$ & $x$ & & Serdyuchenko et al. (2014) \\
\hline HONO & & & $x$ & & Stutz et al. (2000) \\
\hline $\mathrm{BrO}$ & & & $x$ & & Fleischmann (2004) \\
\hline \multirow[t]{3}{*}{$\mathrm{NO}_{2}$} & $293 \mathrm{~K}$ & $1 \times 10^{16}$ molec cm $^{-2}$ & $x$ & & Vandaele et al. (1998) \\
\hline & & & $x$ & & Linear and square terms according to Puksite et al. (2010) \\
\hline & $220 \mathrm{~K}$ & $1 \times 10^{16}$ molec cm $^{-2}$ & $x$ & & \\
\hline Additive polynomial degree & & & 1 & & e.g. Peters et al. (2017) \\
\hline
\end{tabular}

* Water vapour absorption around $363 \mathrm{~nm}$ was not considered for the calculation and analysis of synthetic spectra.

tions for shift and squeeze are linearly independent, which can be favourable in terms of numerical stability.

\subsection{Results}

The variation of the observed spectral shifts of the measurement spectrum during 16 June 2013 is less than 4 pm, as can be seen from Fig. 2. This translates to a spectral shift of less than $0.3 \mathrm{pm} \mathrm{h}^{-1}$ or less than $0.06 \mathrm{pm}$ per elevation angle sequence. This accuracy allows the shift to be distinguished due to the tilt effect (up to $2 \mathrm{pm}$ ) within each elevation angle sequence from instrumental instabilities. The resulting correlation of measured shift and calculated shift determined from the DOAS polynomial is shown for the complete data set (June and July 2013) in Fig. 4 evaluated relative to the next zenith Fraunhofer reference spectrum. The shift due to the tilt effect was calculated from the DOAS polynomial using Eq. (13). As the shift varies with wavelength, we used the average shift calculated on an equidistant grid of $0.1 \mathrm{~nm}$ within the fit interval.

A correlation coefficient $R^{2}=0.83$ and slope of $0.95 \pm$ 0.02 was observed. The $y$ axis intercept of the fitted polynomial was small and amounted to $0.05 \mathrm{pm}$, which is less than $1 / 1000$ of the spectral width of a detector pixel. The small deviation of the fitted slope of the correlation from unity can result from a slightly varying instrument function width within the fit interval $(<2 \%$, estimated from widths of recorded mercury emission line spectra) and effective weighting of the shift at different wavelengths due to variable depth of the Fraunhofer lines (estimated from tilt effect cal- culations of spectra with and without weighting due to Fraunhofer lines to be less than $3 \%$ ). The average measurement error of the shift (estimated by twice the fit error following Stutz and Platt, 1996) amounts to $0.03 \mathrm{pm}(<1.5 \%)$. Furthermore the instrument function of the spectrometer used here is not exactly Gaussian.

Having shown that the shifts are mostly caused by the tilt effect, this allows the measured shift of the reference spectrum for the shift to be corrected by the tilt effect to obtain the instrumental shift at higher precision, also during unsupervised field measurements and without the need for calibration lamps. This is also shown in Fig. 2. The resulting instrumental shift is stable until about 09:00 UTC (with a standard deviation of less than $0.1 \mathrm{pm}$ ), a time after which the room temperature changed, probably as the door was opened and the temperature outside the instrument started changing. The gap in measurement data around noon is caused by a restart of the measurement routine. As the temperature stabilization routine was also restarted, the gap is followed by a shift in the spectral calibration of $0.4 \mathrm{pm}$, as the temperature control needed a few minutes to stabilize. This effect would not have been as clearly visible without correction for the tilt effect.

For an individual spectrum recorded at an elevation angle of $2^{\circ}$ the fit results are shown in Table 2 using a reference spectrum recorded in the same elevation angle sequence at an elevation angle of $90^{\circ}$. Here six cases are distinguished with different numbers of free parameters for shift, squeeze and the explicitly calculated tilt-effect correction spectrum according to Eq. (19). For the calculation of the tilt-effect cor- 
Table 2. DOAS fit results from 16 June 2013 at 04:46 UTC for a spectrum at $2^{\circ}$ elevation for different settings of the spectral shift and squeeze of the reference spectrum (see second row) and with and without a tilt effect correction spectrum marked by crosses in the first row (Sect. 5.2). To minimize photon shot noise, four subsequent elevation angle sequences were co-added. Values in round brackets denote fixed values for shift and squeeze. Fit residuals and a fit of the correction spectrum are shown in Fig. 3. The average shift within the fit interval due to the tilt effect calculated from the DOAS polynomial itself amounts to $1.14 \mathrm{pm}$ and a squeeze of $1+8 \times 10^{-7}$, which could not be resolved from the measurement data. The row named " $\sigma_{\text {fit }}$ " lists the respective fit errors of the obtained differential slant column densities (dSCDs) from the DOAS fit (squeeze definition from DOASIS, $\lambda_{0}=\lambda_{\min }$; see Eq. 17).

\begin{tabular}{|c|c|c|c|c|c|c|}
\hline Case & 1 & 2 & 3 & 4 & 5 & 6 \\
\hline Free shift parameters & shift, squeeze & shift & none & corr. spectrum & $\begin{array}{l}\text { corr. spectrum } \\
\text { and shift }\end{array}$ & none \\
\hline Tilt effect correction spectrum & & & & $x$ & & $x$ \\
\hline Shift (pm) & $1.1 \pm 0.1$ & $0.99 \pm 0.04$ & (0) & (0) & $0.019 \pm 0.04$ & (0) \\
\hline Squeeze & $1.00 \pm 3.4 \times 10^{-6}$ & (1) & (1) & (1) & (1) & (1) \\
\hline $\operatorname{Rms}\left[10^{-4}\right]$ & 2.83 & 2.84 & 4.30 & 2.79 & 2.79 & 2.82 \\
\hline dSCD HONO $\left[1 \times 10^{14}\right.$ molec cm $\left.^{-2}\right]$ & 2.64 & 2.48 & 0.18 & 1.99 & 2.01 & 2.03 \\
\hline$\sigma_{\text {fit }}$ HONO $\left[1 \times 10^{14} \mathrm{molec} \mathrm{cm}^{-2}\right]$ & 2.46 & 2.47 & 3.67 & 2.39 & 2.44 & 2.46 \\
\hline
\end{tabular}
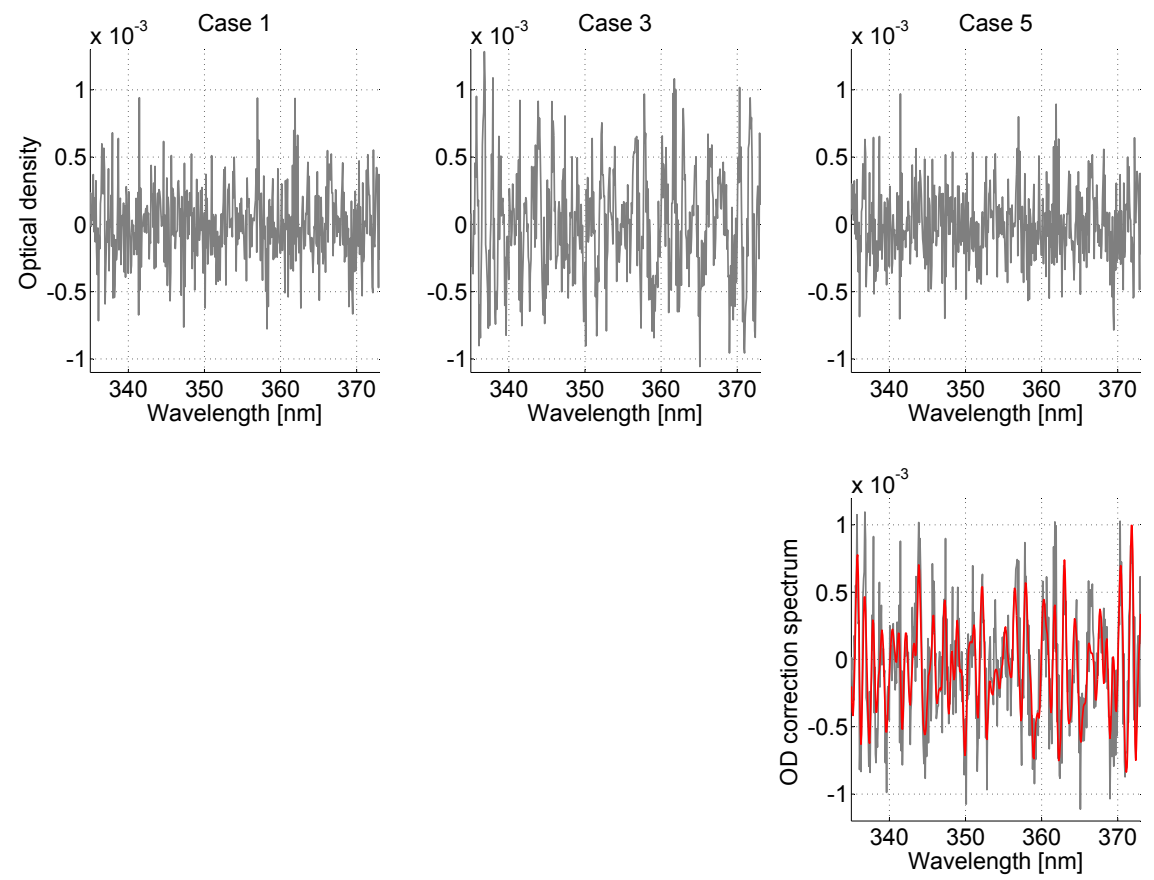

Figure 3. Corresponding plots of fit residuals to the cases 1, 3 and 5 from Table 2 and the tilt-effect correction spectrum for case 5. The tilt-effect correction spectrum is shown in red; the sum of it and the residual are shown in grey.

rection spectrum we used a DOAS polynomial obtained from a fit without considering the tilt effect (see also Sect. 5.2).

\section{The tilt effect in synthetic spectra}

Additionally the tilt effect is demonstrated for synthetic spectra in order to exclude any instrumental influences.

\subsection{Calculation of synthetic spectra}

All simulations were conducted with the radiative transfer model SCIATRAN (Rozanov et al., 2014), version 3.6.0 (3 December 2015). SCIATRAN was operated in raman mode to simulate intensities of scattered sunlight in Mainz, Germany $\left(49.99^{\circ} \mathrm{N}, 8.23^{\circ} \mathrm{E}\right)$, including the effect of rotational Raman scattering in the Earth's atmosphere. The scalar radiative transfer problem was solved in a pseudo-spherical atmosphere (i.e. the solar beam is treated in spherical geom- 


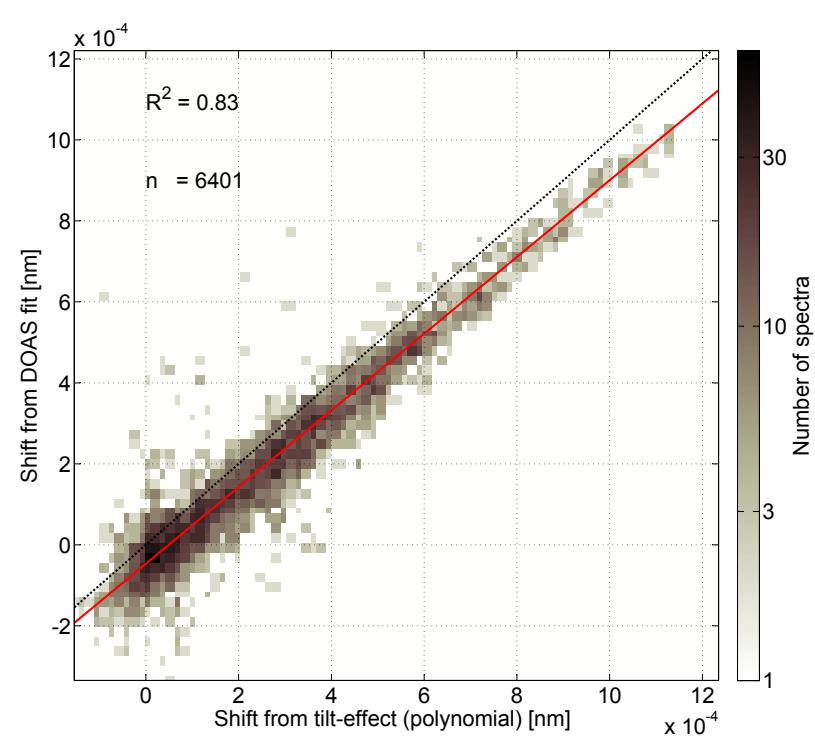

Figure 4. Correlation of the shift determined from the DOAS polynomial according to the tilt effect and measured relative shift of the measurement spectrum to the following zenith sky spectrum (in order to minimize the influence of instrumental instabilities). To reduce the scatter of the data points further, four subsequent elevation angle sequences were co-added.

etry, while the scattered or reflected beam is treated in planeparallel geometry) using the discrete ordinate method. The simulations from 330 to $395 \mathrm{~nm}$ were conducted with $0.01 \mathrm{~nm}$ spectral sampling, and Raman lines were calculated using the forward-adjoint approach and binned to the $0.01 \mathrm{~nm}$ wavelength grid (Rozanov and Vountas, 2014).

Absorption by the trace gases, ozone $\left(\mathrm{O}_{3}\right)$, nitrogen dioxide $\left(\mathrm{NO}_{2}\right)$, formaldehyde $(\mathrm{HCHO})$, bromine oxide $(\mathrm{BrO})$, nitrous acid (HONO), and by the $\mathrm{O}_{2}-\mathrm{O}_{2}$ collision complex $\left(\mathrm{O}_{4}\right)$ was considered. The respective cross-section references are the ones also used for analysis in Table 1. Aerosols were assumed to be mostly scattering, having an optical depth (AOD) of 0.135 , an asymmetry factor of 0.68 , and a single scattering albedo (SSA) of 0.94 . All aerosol parameters were assumed to be constant over the whole wavelength range.

The simulated observation geometry was similar to the measurement sequences as described in Sect. 3.2. A more detailed description, also of the concentration height profiles, can be found in Wang et al. (2017).

Water vapour absorption according to Lampel et al. (2017) and Polyansky et al. (2017) was not considered for the synthetic spectra but was compensated in the measured data. A detailed analysis can be found in Wang et al. (2017).

\subsection{Results}

The spectral analysis was performed in analogy to Sect. 3.3. The absorption of water vapour in the UV was not consid- ered for the calculation of the spectra and thus also not in the spectral analysis.

The synthetic spectra represent measurements of an ideal instrument without any changes of the wavelength calibration due to external influences. Therefore, the initial expectation of the analysis of the synthetic spectra was that no shift is needed in the spectral analysis between reference spectrum and measurement spectrum. However, as described in Sect. 2, some spectral shift was found and needed to be compensated for.

Fits with an rms of more than $4 \times 10^{-4}$ during twilight were filtered out, as saturation and radiative transfer effects of stratospheric ozone absorption increased the residuals of the fits significantly and have the potential to modify the calculated shift values. The correlation of calculated and fitted shift for the remaining 120 spectra due to the tilt effect was very good, with $R^{2}=0.9993$. Shifts of up to $1.2 \mathrm{pm}$ due to the tilt effect were found. The shift from the DOAS fit was about $2.1 \%$ larger than from the calculation of the tilt effect. However, the average measurement error of the shift amounts to $0.02 \mathrm{pm}$ and is thus of similar magnitude.

The small discrepancy could be also caused by the fact that the influence of rotational Raman scattering is calculated differently in DOASIS (according to Bussemer, 1993; Platt and Stutz, 2008 from the convolved, synthetic spectrum itself) and SCIATRAN (according to Rozanov and Vountas, 2014 at the higher spectral resolution of $0.01 \mathrm{~nm}$ before convolution).

Overall the very good agreement of theoretically expected and calculated spectral shifts also shows the validity of the derivation of the tilt effect.

\section{Discussion - correction of the tilt effect}

Even for a perfect MAX- or zenith sky DOAS instrument (as shown in Sect. 4), the tilt effect needs to be considered and corrected. Typically it is corrected by allowing a shift between measurement spectra and reference spectra. As the shift at each wavelength depends on the derivative of the broadband spectral dependence, which is usually corrected by the DOAS polynomial, an additional squeeze (and higher orders of the spectral shift) of the measurement spectrum can be necessary, depending on the desired magnitude of the residual. This is discussed in Sect. 5.1.

The spectral shift depends on the spectral resolution of the instrument (see Eq. 13). In fact it is proportional to the square of the spectral resolution.

Another approach is to calculate the effective shift spectrum from the explicit calculation of the commutator of polynomial and convolution, or in other words the difference between $p(\lambda) s(\lambda)$ and $t(\lambda)$. This approach is discussed in Sect. 5.2. 


\subsection{Shift and squeeze}

The apparent change in the wavelength determination due to the tilt effect can be determined from the DOAS polynomial using Eq. (13). The shift, squeeze and higher-order parameters can then be determined by a polynomial fit of $\Delta \lambda(\lambda)$ using the QDOAS definition of $\lambda_{0}$ of squeeze and higher orders (see Eq. 17). For each of the parameters of the polynomial (corresponding to shift, squeeze, quadratic squeeze etc.), the maximum shift inside the fit range can be determined and can then be used for estimating the residual structure which is caused by the tilt effect. This shift, converted to the corresponding optical depth, is shown in Fig. 5. For typical applications (FWHM $\left.=0.6 \mathrm{~nm}, \mathrm{rms}>1 \times 10^{-4}\right)$, it is therefore sufficient to allow shift and squeeze between measurement spectra and reference spectra in order to correct for this effect. The conversion factor $\alpha_{\mathrm{OD}}$ from shift to peakto-peak optical density within the fitting interval was determined from the pseudo-absorber of the spectral shift within the fit interval for the given spectral resolution of the instrument and amounted in this case to $1.5 \mathrm{~nm}^{-1}$.

$\alpha_{\mathrm{OD}}=2 \times\left|\frac{\partial s(\lambda)}{\partial \lambda} \frac{1}{s(\lambda)}\right|_{\max }$

\subsection{Tilt-effect correction spectrum}

For a known DOAS polynomial $d(\lambda)$, a correction spectrum $c(\lambda)$ can be calculated to compensate for the tilt effect. This implies that an iterative fit process is performed and thus means higher computational costs. The correction spectrum $c(\lambda)$ is the difference between two synthetic sun spectra calculated from a highly resolved solar atlas, one where the attenuation with the $p(\lambda)=e^{-d(\lambda)}$ in intensity space is applied before the convolution operation and one where it is applied after:

$c(\lambda)=p(\lambda) s(\lambda)-t(\lambda)$.

To use it in the DOAS fit as a pseudo-absorber (PA), it can be converted (in a first-order approximation) to optical density space by division with $s(\lambda)$

$c_{\mathrm{PA}}(\lambda)=\frac{c(\lambda)}{s(\lambda)}$.

This correction spectrum, introduced in the fit results shown in Table 2, was indeed found in the spectral fit and reduced the shift of the reference spectrum from $1.1 \pm 0.1 \mathrm{pm}$ (case 2) to $0.019 \pm 0.04 \mathrm{pm}$ (case 5). As the calculation from Eq. (19) also provides the absolute magnitude of the effect, this correction spectrum does not even need to be fitted as in previous publications but can be applied directly (case 6). This can, if the instrument itself is stable, potentially reduce the degrees of freedom of the fit and thus result in lower detection limits. This could, however, not be observed here for measurement data.
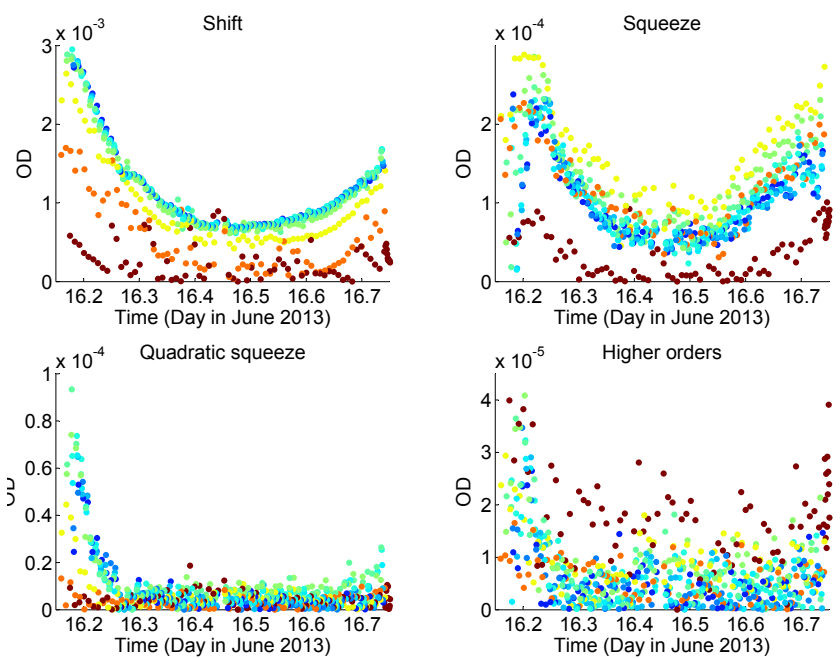

Figure 5. Peak-to-peak optical density caused by shift, squeeze and higher-order squeeze due to the tilt effect, using the data set from Fig. 2. The shift due to the tilt effect was calculated from the DOAS polynomial for the corresponding mean wavelength of each pixel within the fit interval. Then a third-order polynomial was fitted to this data to calculate the corresponding shift, squeeze and higherorder terms and thus the corresponding peak-to-peak ODs caused by the tilt effect. It can be seen that shift and squeeze already compensate for most of the effect. The colour scale is the same as in Fig. 2.

The DOAS polynomial can be determined with sufficient precision without correcting the tilt effect, as a small spectral shift can be represented via Taylor expansion by an individual spectrum, which is dominated by narrowband contributions (Beirle et al., 2013) as it is defined via the derivative with respect to wavelength of the respective spectrum. To test this, the DOAS polynomial was determined for the spectrum shown in Table 2. This polynomial was used to calculate the correction spectrum. The absolute magnitude of the resulting DOAS polynomials with and without correcting for the tilt effect differed relative to each other by up to $3 \%$. Calculating the correction spectrum from the second DOAS polynomial results in a second correction spectrum, which differs absolutely with an OD of $6 \times 10^{-5}$. Therefore further iterations of the fitting process are not needed in this case.

Note that this approach might need to also consider strong absorbers present in the observed spectra, which are not present in the solar atlas spectrum. This can play a role in ozone and sulfur dioxide absorption in the UV range and for strong absorbers such as $\mathrm{H}_{2} \mathrm{O}$ and $\mathrm{O}_{2}$ in the red and near-IR spectral range. A potential disadvantage is that this calculation requires knowledge of the exact instrument slit function, which is implicitly included in the first approach (Sect. 5.1 and see also Sect. 5.3). As the spectral shift of the instrument also needs to be accounted for often, shift and squeeze need to be implemented in any case, which can make the calcu- 
lation of explicit correction spectra in most cases obsolete. This choice depends on the desired precision of the result for a very small rms (compare Fig. 5).

Apart from the tilt-effect-induced shift, the correction spectra calculated using the DOAS polynomial also includes the effect of the squeeze parameter and higher orders. Therefore a correction spectrum needs to be calculated corresponding to each fit. As seen from Fig. 5 applying shift and squeeze is sufficient for most applications, but calculation of the correction spectrum can reduce the impact of the tilt effect even further, as seen in Table 2. Here the rms of the default fit using shift and squeeze of the reference spectrum (1) is reduced by using an explicitly calculated correction spectrum slightly by $1 \%$ (4), even though the number of degrees of freedom of the fit stayed constant (cases $1+5$ ). When only the correction spectrum was used, and the shift fixed to zero, assuming no shift between Fraunhofer reference and measurement spectrum (cases $1+4$ ), the rms is the same, but the HONO fit error is reduced.

\subsection{The influence of the instrument slit function}

As shown in Eq. (13) for the case of a Gaussian instrument response function, the spectral shift depends on the spectral resolution of the instrument, in fact it is proportional to the square to the spectral resolution. A real instrument function $h\left(\lambda_{0}, \lambda\right)$ is in general not a Gaussian function but can be approximated by $N$ Gaussian functions of different widths $\sigma_{i}$ shifted by $\Delta \lambda_{0 i}$ and weighted by $w_{i}$, as it is typically also measured at finite spectral resolution.

$h\left(\lambda_{0}, \lambda\right)=\sum_{i}^{N} w_{i} g_{\sigma_{i}}\left(\lambda_{0}+\Delta \lambda_{0 i}, \lambda\right)$

As summing and convolution are interchangeable, Eq. (1) can be written as a sum over different $s_{i}(\lambda)=k(\lambda) \otimes g_{\sigma_{i}}\left(\lambda_{0}+\right.$ $\left.\Delta \lambda_{0 i}, \lambda\right)$ using Eq. (21). To these Gaussian instrument functions the derivation of the tilt effect applies individually. However, as the derivative with respect to $\lambda$ in the Taylor series for the spectral shift in Eq. (4) also commutes with the sum, the shift calculated from $s(\lambda)$ also correctly compensates for the tilt effect for non-Gaussian instrument functions.

\subsection{Instrument slit function changes due to tilt effect}

As already pointed out for Eq. (7), the squeeze and secondorder effects of the tilt effect also lead to a slight modification of the effective instrument slit function's shape, apart from the spectral shift. Based on the DOAS polynomials obtained from the fits of measurements from 16 June (compare Fig. 2) and using an initial Gaussian instrument slit function, the effective instrument slit function was numerically calculated and fitted again with a Gaussian function. The first-order tilteffect shift was reproduced within $2 \times 10^{-8} \mathrm{~nm}$. The relative width of the instrument function varied by up to $5 \times 10^{-4} \%$.
For an absorber with a differential OD of unity, this results in an OD of less than $5 \times 10^{-6}$ and is therefore negligible.

\subsection{Pixel-wavelength calibration of spectra}

As the tilt effect will influence all spectra recorded at low resolution, it will also have an effect on the spectral calibration of scattered sunlight spectra, if done by fitting it to a highresolution solar atlas, as e.g. Chance and Kurucz (2010). As will be shown in Sect. 5.6, the effect on retrieved trace gases is typically negligible, as the expected shifts due to the tilt effect are also here of the order of less than a few pm.

Note that also other calibration methods, as e.g. the calibration using line emission spectra, have uncertainties: If the position of the emission lines is determined by fitting Gaussian peaks, the fit error of the centre of the peak also typically amounts to $2-3 \mathrm{pm}$, as the shape of the observed emission line is rarely Gaussian (e.g. Liu et al., 2015; Beirle et al., 2017). The width of a single pixel for the measurements shown above is typically $60 \mathrm{pm}$ or larger. The variation between different mercury emission lamps is about $0.07 \mathrm{pm}$ and thus significantly smaller than the tilt effect itself (Sansonetti and Reader, 2001).

The centre of mass of an emission peak can be more accurately determined when the emission peaks are not undersampled.

\subsection{The impact of the tilt effect on the spectral retrieval of trace-gases}

The impact of the tilt effect on the spectral retrieval of trace gases is two-fold: if the tilt effect is not corrected for, the remaining residual structures can cause deviations for retrieved trace gases. The shift induced on the measurement spectrum is the same as for the absorbers, as similar considerations apply to the convolution of trace gases as to the convolution of the Fraunhofer spectrum. However, if the shift of the trace gases is not determined from the fit but from a fit of the Fraunhofer reference spectrum to a solar atlas (typically with a different tilt or colour indices), small shifts of the order of a few picometres can occur, which are not the same for the absorbers.

Using a pseudo-absorber for the spectral shift of $\mathrm{NO}_{2}$ $\partial / \partial \lambda \sigma_{\mathrm{NO}_{2}}(\lambda)$, we obtain a residual OD for a shift of $2 \mathrm{pm}$ due to the tilt effect of the $\mathrm{NO}_{2}$ absorption cross section of $0.2 \%$. Thus a $1.5 \%$ differential absorption by $\mathrm{NO}_{2}$, which corresponds to a differential slant column density (dSCD) of $1 \times 10^{17} \mathrm{molec} \mathrm{cm}^{-2}$, can result in a systematic residual structure due to the tilt effect of $3 \times 10^{-4}$ ( $2 \%$ of the original absorption), which is often acceptable.

For the case of HONO and a spectrum with an apparent shift due to the tilt effect of $1 \mathrm{pm}$, the results are shown in Table 2. It becomes clear that the overall influence of the tilt effect on the retrieved HONO dSCDs is small and within the measurement error in this case, for this absorber and for 
this instrument. However, as the residual rms and thus the fit error are significantly reduced, the correction of this effect is crucial for a correct determination of measurement errors and detection limits (cf. e.g. Stutz and Platt, 1996). If the shape of the structures caused by the tilt effect shows more similarities with an absorber, the changes in its dSCDs might, however, be larger. This depends on the fitting interval, spectral resolution and the respective absorber and cannot be answered in general.

\section{Conclusions}

Based on a theoretical analysis as well as on measured and simulated scattered sunlight spectra, we have shown that the tilt effect can cause artificial shifts and enhanced residuals, which are introduced by the fact that any modification of the broadband spectral variation of a spectrum (e.g. caused by atmospheric scattering processes) does not commute with the convolution with the instrument slit function. Thus an effective shift between measurement and reference spectra can be observed. This effect is called the tilt effect according to Sioris et al. (2003). In the context of limb satellite observations, this effect was mathematically described by Rozanov et al. (2011). We showed that the spectral shift due to this effect is proportional to the square of the instrument resolution $\sigma$ and the slope of the broadband spectral shape. It can be described by the so-called DOAS polynomial, which accounts for broadband spectral differences between the measured spectrum and the Fraunhofer reference spectrum (e.g. caused by Mie and Rayleigh scattering and broadband absorptions). In contrast to previous publications (e.g. Sioris et al., 2003), it is not directly connected to spectral undersampling (Chance et al., 2005) and is not restricted to a certain wavelength range. It affects any medium-resolution spectroscopic application where the spectral evaluation involves a step in which the convolution and effects like scattering are commuted, which leads to a broadband variation of the shape of the spectrum. Lab measurements of trace gas absorptions are, however, often done at higher spectral resolution, which minimizes the apparent shift of the tilt effect due to the relation shown in Eq. (13).

A shift between measurement spectra and reference spectra is typically allowed for in DOAS retrievals and motivated by instrumental instabilities. We show that the shift caused by the tilt effect is significantly larger than typical instrument shifts within one elevation sequence and that the main reason to allow for this shift is eventually the tilt effect. For measured as well as for simulated spectra a good correlation between fitted and calculated shifts is found due to the tilt effect.

For ground-based passive DOAS instruments with a spectral resolution of $0.6 \mathrm{~nm}$, we find apparent spectral shifts of more than $1 \mathrm{pm}$ due to the tilt effect. This shift can result in residual optical depths of $2.5 \times 10^{-3}$ if not corrected for. This will increase the calculated fit and measurement errors and can also lead to deviations of retrieved dSCDs, depending on the settings of the spectral retrieval and the instrument's properties. For DOAS fits with a residual rms of more than $10^{-4}$, we estimate that the tilt effect can be compensated for by allowing for a shift and squeeze term. For DOAS fits with a residual $\mathrm{rms}$ of less than $10^{-4}$, which can be obtained by co-adding a large number of spectra, higher-order terms for the parameterization of the wavelength shift might be necessary. The shift due to the tilt effect is typically not constant with respect to wavelength $\lambda$ within the fit intervals, as it is proportional to the derivative of the so-called DOAS polynomial (Eq. 13). For observation geometries which show larger differences in colour indices, such as satellite limb observations, such corrections might even be necessary if the requirements on the magnitude of the residual are less strict. As the spectral shift due to the tilt effect can be calculated from the DOAS polynomial, the remaining observed spectral shift can be attributed to instrumental properties and it can thus be used for monitoring purposes.

Alternatively, using the known instrument function, correction spectra can be explicitly calculated for a given DOAS polynomial or approximated from a given difference in colour indices between measurement spectra and reference spectra, similarly to suggestions in previous publications.

The effect is generally present for spectroscopic measurements at medium spectral resolution with wavelengthdependent attenuation. Therefore the same effect can be expected for active measurements (e.g. cavity-enhanced or long-path DOAS measurements).

Data availability. The spectra used for the data evaluation in this paper (about $420 \mathrm{MB}$ ) can be obtained on request from the authors.

Competing interests. The authors declare that they have no conflict of interest.

Acknowledgements. We thank the MAD-CAT team for support during the MAD-CAT campaign. We thank Klaus Pfeilsticker and Alexey Rozanov for helpful comments during the preparation of the manuscript. We thank EnviMeS/Airyx for providing the MAX-DOAS instrument during the MAD-CAT campaign.

The article processing charges for this open-access publication were covered by the Max Planck Society.

Edited by: Michel Van Roozendael

Reviewed by: three anonymous referees 


\section{References}

Beirle, S., Sihler, H., and Wagner, T.: Linearisation of the effects of spectral shift and stretch in DOAS analysis, Atmos. Meas. Tech., 6, 661-675, https://doi.org/10.5194/amt-6-661-2013, 2013.

Beirle, S., Lampel, J., Lerot, C., Sihler, H., and Wagner, T.: Parameterizing the instrumental spectral response function and its changes by a super-Gaussian and its derivatives, Atmos. Meas. Tech., 10, 581-598, https://doi.org/10.5194/amt-10-5812017, 2017.

Burton, M. R. and Sawyer, G. M.: iFit: An intensity-based retrieval for $\mathrm{SO}_{2}$ and $\mathrm{BrO}$ from scattered sunlight ultraviolet volcanic plume absorption spectra, Atmos. Meas. Tech. Discuss., https://doi.org/10.5194/amt-2015-380, in review, 2016.

Bussemer, M.: Der Ring-Effekt: Ursachen und Einfluß auf die spektroskopische Messung stratosphärischer Spurenstoffe, Diploma thesis, Heidelberg University, Heidelberg, Germany, 1993.

Chance, K. and Kurucz, R.: An improved high-resolution solar reference spectrum for earth's atmosphere measurements in the ultraviolet, visible, and near infrared, J. Quant. Spectrosc. Ra., 111, 1289-1295, https://doi.org/10.1016/j.jqsrt.2010.01.036, 2010.

Chance, K., Kurosu, T. P., and Sioris, C. E.: Undersampling correction for array detector-based satellite spectrometers, Appl. Optics, 44, 1296-1304, https://doi.org/10.1364/AO.44.001296, 2005.

Danckaert, T., Fayt, C., Roozendael, M. V., Smedt, I. D., Letocart, V., Merlaud, A., and Pinardi, G.: QDOAS Software user manual, available at: http://uv-vis.aeronomie.be/software/QDOAS/ QDOAS_manual.pdf (last access: 30 November 2017), 2012.

Fleischmann, O.: New ultraviolet absorption cross-sections of $\mathrm{BrO}$ at atmospheric temperatures measured by time-windowing Fourier transform spectroscopy, J. Photoch. Photobio. A, 168, 117-132, 2004.

Haley, C. S., Brohede, S. M., Sioris, C. E., Griffioen, E., Murtagh, D. P., McDade, I. C., Eriksson, P., Llewellyn, E. J., Bazureau, A., and Goutail, F.: Retrieval of stratospheric $\mathrm{O}_{3}$ and $\mathrm{NO}_{2}$ profiles from Odin Optical Spectrograph and Infrared Imager System (OSIRIS) limb-scattered sunlight measurements, J. Geophys. Res.-Atmos., 109, D16303, https://doi.org/10.1029/2004JD004588, 2004.

Hönninger, G. and Platt, U.: Observations of $\mathrm{BrO}$ and its vertical distribution during surface ozone depletion at Alert, Atmos. Environ., 36, 2481-2489, 2002.

Kraus, S.: DOASIS - A Framework Design for DOAS, Dissertation, Heidelberg University, Heidelberg, Germany, 2006.

Krecl, P., Haley, C. S., Stegman, J., Brohede, S. M., and Berthet, G.: Retrieving the vertical distribution of stratospheric $\mathrm{OClO}$ from Odin/OSIRIS limb-scattered sunlight measurements, Atmos. Chem. Phys., 6, 1879-1894, https://doi.org/10.5194/acp-61879-2006, 2006.

Lampel, J., Frieß, U., and Platt, U.: The impact of vibrational Raman scattering of air on DOAS measurements of atmospheric trace gases, Atmos. Meas. Tech., 8, 3767-3787, https://doi.org/10.5194/amt-8-3767-2015, 2015.

Lampel, J., Pöhler, D., Polyansky, O. L., Kyuberis, A. A., Zobov, N. F., Tennyson, J., Lodi, L., Frieß, U., Wang, Y., Beirle, S., Platt, U., and Wagner, T.: Detection of water vapour absorption around $363 \mathrm{~nm}$ in measured atmospheric absorption spectra and its effect on DOAS evaluations, Atmos. Chem. Phys., 17, 1271-1295, https://doi.org/10.5194/acp-17-1271-2017, 2017.
Liu, C., Liu, X., Kowalewski, M. G., Janz, S. J., González Abad, G., Pickering, K. E., Chance, K., and Lamsal, L. N.: Characterization and verification of ACAM slit functions for tracegas retrievals during the 2011 DISCOVER-AQ flight campaign, Atmos. Meas. Tech., 8, 751-759, https://doi.org/10.5194/amt-8751-2015, 2015.

Lübcke, P., Lampel, J., Arellano, S., Bobrowski, N., Dinger, F., Galle, B., Garzón, G., Hidalgo, S., Chacón Ortiz, Z., Vogel, L., Warnach, S., and Platt, U.: Retrieval of absolute $\mathrm{SO}_{2}$ column amounts from scattered-light spectra: implications for the evaluation of data from automated DOAS networks, Atmos. Meas. Tech., 9, 5677-5698, https://doi.org/10.5194/amt-9-5677-2016, 2016.

McLinden, C. A. and Haley, C. S.: Odin/OSIRIS observations of stratospheric $\mathrm{NO}_{3}$ through sunrise and sunset, Atmos. Chem. Phys., 8, 5529-5534, https://doi.org/10.5194/acp-8-5529-2008, 2008.

McLinden, C. A., Haley, C., Lloyd, N., Hendrick, F., Rozanov, A., Sinnhuber, B.-M., Goutail, F., Degenstein, D., Llewellyn, E., Sioris, C., Van Roozendael, M., Pommereau, J. P., Lotz, W., and Burrows, J. P.: Odin/OSIRIS observations of stratospheric BrO: Retrieval methodology, climatology, and inferred Bry, J. Geophys. Res.-Atmos., 115, D15308, https://doi.org/10.1029/2009JD012488, 2010.

Meller, R. and Moortgat, G. K.: Temperature dependence of the absorption cross sections of formaldehyde between $223 \mathrm{~K}$ and $323 \mathrm{~K}$ in the wavelength range 225$375 \mathrm{~nm}$, J. Geophys. Res.-Atmos., 105, 7089-7101, https://doi.org/10.1029/1999JD901074, 2000.

Ortega, I., Koenig, T., Sinreich, R., Thomson, D., and Volkamer, R.: The CU 2-D-MAX-DOAS instrument - Part 1: Retrieval of 3-D distributions of $\mathrm{NO}_{2}$ and azimuth-dependent OVOC ratios, Atmos. Meas. Tech., 8, 2371-2395, https://doi.org/10.5194/amt8-2371-2015, 2015.

Peters, E., Pinardi, G., Seyler, A., Richter, A., Wittrock, F., Bösch, T., Van Roozendael, M., Hendrick, F., Drosoglou, T., Bais, A. F., Kanaya, Y., Zhao, X., Strong, K., Lampel, J., Volkamer, R., Koenig, T., Ortega, I., Puentedura, O., Navarro-Comas, M., Gómez, L., Yela González, M., Piters, A., Remmers, J., Wang, Y., Wagner, T., Wang, S., Saiz-Lopez, A., García-Nieto, D., Cuevas, C. A., Benavent, N., Querel, R., Johnston, P., Postylyakov, O., Borovski, A., Elokhov, A., Bruchkouski, I., Liu, H., Liu, C., Hong, Q., Rivera, C., Grutter, M., Stremme, W., Khokhar, M. F., Khayyam, J., and Burrows, J. P.: Investigating differences in DOAS retrieval codes using MAD-CAT campaign data, Atmos. Meas. Tech., 10, 955-978, https://doi.org/10.5194/amt-10-9552017, 2017.

Platt, U. and Stutz, J.: Differential optical absorption spectroscopy, Springer, Berlin, Heidelberg, Germany, 2008.

Polyansky, O. L., Kyuberis, A. A., Lodi, L., Tennyson, J., Ovsyannikov, R. I., and Zobov, N.: ExoMol molecular line lists XX: high accuracy computed line list for hot $\mathrm{H}_{2}{ }^{16} \mathrm{O}$, Mon. Not. R. Astron. Soc., in preparation, 2017.

Puksīte, J., Kühl, S., Deutschmann, T., Platt, U., and Wagner, T.: Extending differential optical absorption spectroscopy for limb measurements in the UV, Atmos. Meas. Tech., 3, 631-653, https://doi.org/10.5194/amt-3-631-2010, 2010.

Rozanov, A., Kühl, S., Doicu, A., McLinden, C., Puķīe, J., Bovensmann, H., Burrows, J. P., Deutschmann, T., Dorf, M., Goutail, 
F., Grunow, K., Hendrick, F., von Hobe, M., Hrechanyy, S., Lichtenberg, G., Pfeilsticker, K., Pommereau, J. P., Van Roozendael, M., Stroh, F., and Wagner, T.: BrO vertical distributions from SCIAMACHY limb measurements: comparison of algorithms and retrieval results, Atmos. Meas. Tech., 4, 1319-1359, https://doi.org/10.5194/amt-4-1319-2011, 2011.

Rozanov, V. V. and Vountas, M.: Radiative transfer equation accounting for rotational Raman scattering and its solution by the discrete-ordinates method, J. Quant. Spectrosc. Ra., 133, 603618, https://doi.org/10.1016/j.jqsrt.2013.09.024, 2014.

Rozanov, V. V., Rozanov, A. V., Kokhanovsky, A. A., and Burrows, J. P.: Radiative transfer through terrestrial atmosphere and ocean: Software package SCIATRAN, J. Quant. Spectrosc. Ra., 133, 13-71, https://doi.org/10.1016/j.jqsrt.2013.07.004, 2014.

Sansonetti, C. J. and Reader, J.: Spectrum and Energy Levels of Singly-Ionized Mercury (Hg II), Phys. Scripta, 63, 219, doi10.1238/Physica.Regular.063a00219, 2001.

Sarkissian, A., Pommereau, J., and Goutail, F.: Identification of polar stratospheric clouds from the ground by visible spectrometry, Geophys. Res. Lett., 18, 779-782, 1991.

Serdyuchenko, A., Gorshelev, V., Weber, M., Chehade, W., and Burrows, J. P.: High spectral resolution ozone absorption crosssections - Part 2: Temperature dependence, Atmos. Meas. Tech., 7, 625-636, https://doi.org/10.5194/amt-7-625-2014, 2014.

Sioris, C. E., Haley, C. S., McLinden, C. A., von Savigny, C., McDade, I. C., McConnell, J. C., Evans, W. F., Lloyd, N. D., Llewellyn, E. J., Chance, K. V., Kurosu, T. P., Murtagh, D., Frisk, U., Pfeilsticker, K., Bösch, H., Weidner, F., Strong, K., Stegman, J., and Mégie, G.: Stratospheric profiles of nitrogen dioxide observed by Optical Spectrograph and Infrared Imager System on the Odin satellite, J. Geophys. Res.-Atmos., 108, 4215, https://doi.org/10.1029/2002JD002672, 2003.

Sioris, C. E., Kurosu, T., Martin, R., and Chance, K.: Stratospheric and tropospheric $\mathrm{NO}_{2}$ observed by SCIAMACHY: first results, Adv. Space Res., 34, 780-785, https://doi.org/10.1016/j.asr.2003.08.066, 2004.

Sioris, C. E., Kovalenko, L. J., McLinden, C. A., Salawitch, R. J., Van Roozendael, M., Goutail, F., Dorf, M., Pfeilsticker, K., Chance, K., von Savigny, C., Liu, X., Kurosu, T. P., Pommereau, J.-P., Bösch, H., and Frerick, J.: Latitudinal and vertical distribution of bromine monoxide in the lower stratosphere from Scanning Imaging Absorption Spectrometer for Atmospheric Chartography limb scattering measurements, J. Geophys. Res.Atmos., 111, D14301, https://doi.org/10.1029/2005JD006479, 2006.
Slijkhuis, S., von Bargen, A., Thomas, W., and Chance, K.: Calculation of Undersampling Correction Spectra for DOAS Spectral Fitting, in: Atmospheric Measurements from Space, Vol. 2, 563569, ESAMS'99, European Symposium on Atmospheric Measurements from Space, 18-22 January 1999, Noordwijk, the Netherlands, 1999.

Stutz, J. and Platt, U.: Numerical Analysis and Estimation of the Statistical Error of Differential Optical Absorption Spectroscopy Measurements with Least-Squares methods, Appl. Optics, 35, 6041-6053, 1996.

Stutz, J., Kim, E. S., Platt, U., Bruno, P., Perrino, C., and Febo, A.: UV-visible absorption cross sections of nitrous acid, J. Geophys. Res., 105, 14585, https://doi.org/10.1029/2000JD900003, 2000.

Thalman, R. and Volkamer, R.: Temperature dependent absorption cross-sections of $\mathrm{O}_{2}-\mathrm{O}_{2}$ collision pairs between 340 and $630 \mathrm{~nm}$ and at atmospherically relevant pressure, Phys. Chem. Chem. Phys., 15, 15371-15381, https://doi.org/10.1039/C3CP50968K, 2013.

Vandaele, A., Hermans, C., Simon, P., Carleer, M., Colin, R., Fally, S., Merienne, M., Jenouvrier, A., and Coquart, B.: Measurements of the $\mathrm{NO}_{2}$ absorption cross-section from $42000 \mathrm{~cm}^{-1}$ to $10000 \mathrm{~cm}^{-1}(238-1000 \mathrm{~nm})$ at $220 \mathrm{~K}$ and $294 \mathrm{~K}$, J. Quant. Spectrosc. Ra., 59, 171-184, https://doi.org/10.1016/S00224073(97)00168-4, 1998.

Wagner, T., Deutschmann, T., and Platt, U.: Determination of aerosol properties from MAX-DOAS observations of the Ring effect, Atmos. Meas. Tech., 2, 495-512, https://doi.org/10.5194/amt-2-495-2009, 2009.

Wang, Y., Beirle, S., Hendrick, F., Hilboll, A., Jin, J., Kyuberis, A. A., Lampel, J., Li, A., Luo, Y., Lodi, L., Ma, J., Navarro, M., Ortega, I., Peters, E., Polyansky, O. L., Remmers, J., Richter, A., Puentedura, O., Van Roozendael, M., Seyler, A., Tennyson, J., Volkamer, R., Xie, P., Zobov, N. F., and Wagner, T.: MAX-DOAS measurements of HONO slant column densities during the MAD-CAT campaign: inter-comparison, sensitivity studies on spectral analysis settings, and error budget, Atmos. Meas. Tech., 10, 3719-3742, https://doi.org/10.5194/amt10-3719-2017, 2017.

Wenig, M., Jähne, B., and Platt, U.: Operator representation as a new differential optical absorption spectroscopy formalism, Appl. Optics, 44, 3246-3253, 2005. 